\title{
Impact of levosimendan on brain injury patterns in a lamb model of infant cardiopulmonary bypass
}

\author{
Poongundran Namachivayam ${ }^{1,2}$, Joseph J. Smolich ${ }^{2,3}$, Amy E. Shields', Sandra Rees ${ }^{4}$, Lee Coleman ${ }^{5}$, Steven B. Horton ${ }^{6}$,
} Igor E. Konstantinov $2,3,6$, Daniel J. Penny ${ }^{7}$ and Lara S. Shekerdemian ${ }^{8}$

BACKGROUND: The effects of levosimendan (Levo) on injury patterns in the immature brain following cardiopulmonary bypass (CPB) are unknown.

METHODS: Eighteen 3- to 4-wk-old anesthetized lambs, instrumented with vascular catheters and aortic and right carotid artery flow probes, were allocated to non-CPB, CPB, or $\mathrm{CPB}+$ Levo groups (each $n=6$ ). After $120 \mathrm{~min} C P B$ with $90 \mathrm{~min}$ aortic cross-clamp, CPB animals received dopamine, and CPB+Levo animals both dopamine and Levo, for $4 \mathrm{~h}$. All lambs then underwent brain magnetic resonance imaging, followed by postmortem brain perfusion fixation for immunohistochemical studies.

RESULTS: In CPB lambs, aortic $(P<0.05)$ and carotid artery $(P<0.01)$ blood flows fell by 29 and 30\%, respectively, between 2 and $4 \mathrm{~h}$ after cross-clamp removal but were unchanged in the CPB+Levo group. No brain injury was detectable with magnetic resonance imaging in either CPB or CPB+Levo lambs. However, on immunohistochemical analysis, white matter astrocyte density of both groups was higher than in non-CPB lambs $(P<0.05)$, while white matter microglial density was higher $(P<0.05)$, but markers of cortical oxidative stress were less prevalent in CPB+Levo than CPB lambs.

CONCLUSION: While Levo prevented early postoperative falls in cardiac output and carotid artery blood flow in a lamb model of infant CPB, this was associated with heterogeneous neuroglial activation and manifestation of markers of oxidative stress.

$\mathbf{N}$ eurodevelopmental impairment affects up to one half of all survivors of infant heart surgery, with the spectrum ranging from gross and fine motor deficits, visuospatial difficulties, and impaired cognition to delay in speech and language development (1-5). The mechanisms contributing to brain injury in infants with congenital heart disease are complex and multifactorial, with no consistent improvement in neurodevelopmental outcomes achieved despite major advances in pediatric cardiac surgery and survival. A recent report from the Pediatric Heart Network and the National Heart, Lung, and Blood Institute working group reaffirmed the importance of trials aimed at evaluating novel therapies to address this problem (6).

Younger infants undergoing surgery for congenital heart disease are most susceptible to developing brain injury $(7,8)$. The perioperative interval represents a high-risk period with often extended duration of cardiopulmonary bypass (CPB) at the time of the first surgical procedure, as well as significant circulatory disturbance postoperatively. The avoidance of a low cardiac output (CO) state, which can complicate the postoperative course of a proportion of infants after cardiac surgery and which may contribute to later impairment, is a major focus of postoperative care after infant heart surgery. The PRIMACORP study demonstrated the efficacy of intravenous milrinone in preventing this phenomenon in a cohort of children undergoing intracardiac repairs (9). Our laboratory subsequently demonstrated that the inodilator levosimendan (Levo) (Simdax, Abbott, Botany, Australia) was at least as efficacious, and potentially more so, than milrinone in protecting against a postoperative reduction of $\mathrm{CO}$ in a piglet model of infant CPB (10).

Levo improves cardiovascular performance through sensitization of the myocardium to intracellular calcium, resulting in increased contractility, and through opening of sarcolemmal adenosine triphosphate (ATP)-sensitive $\mathrm{K}^{+}$channels in vascular smooth muscle cells to produce peripheral and coronary vasodilation (10). Mitochondria play a major role in cellular responses to ischemic injury, and the activation of mitochondrial ATP-sensitive $\mathrm{K}^{+}$channels confers neuroprotection in models of $\mathrm{CPB}$ and in models of cerebral ischemia-reperfusion $(11,12)$. More recently, the action of Levo on mitochondrial ATP-sensitive $\mathrm{K}^{+}$channels in the heart has been shown to protect the heart against ischemia-reperfusion injury and to limit myocyte apoptosis (13).

While it has beneficial hemodynamic actions and impacts positively upon cardiovascular performance, whether Levo impacts the development of brain injury related to pediatric

\footnotetext{
'Pediatric Intensive Care Unit, The Royal Children's Hospital, Melbourne, Australia; ${ }^{2}$ Murdoch Children's Research Institute, Melbourne, Australia; ${ }^{3}$ Department of Paediatrics, University of Melbourne, Melbourne, Australia; ${ }^{4}$ Department of Anatomy and Cell Biology, University of Melbourne, Melbourne, Australia; ${ }^{5}$ Department of Radiology, The Royal Children's Hospital, Melbourne, Australia; ${ }^{6}$ Cardiac Surgery, The Royal Children's Hospital, Melbourne, Australia; ${ }^{7}$ Section of Cardiology, Texas Children's Hospital, Houston, Texas; ${ }^{8}$ Section of Critical Care Medicine, Texas Children's Hospital, Houston, Texas. Correspondence: Lara S. Shekerdemian (Issheker@texaschildrens.org) 
$\mathrm{CPB}$ is currently unknown. To address this question, we investigated the effects of Levo on circulatory hemodynamics, brain magnetic resonance imaging (MRI), and brain immunochemistry in a lamb model of infant CPB.

\section{RESULTS}

\section{Blood Gases and Hemodynamics}

Baseline arterial blood gases, mixed venous oxygen saturation $\left(\mathrm{SvO}_{2}\right)$, heart rate, aortic pressure $\left(P_{\mathrm{AO}}\right)$, pulmonary artery pressure $\left(P_{\mathrm{PA}}\right), \mathrm{CO}$, and right carotid artery blood flow $\left(Q_{\mathrm{CA}}\right)$ were similar in all groups (Tables 1 and 2). Compared with baseline, arterial $\mathrm{pH}, \mathrm{PcO}_{2}$, and $\mathrm{Po}_{2}$ at the four post-CPB time points were unchanged in the three groups (data not shown). However, while $\mathrm{SvO}_{2}$ (Table 2) was unaltered in the non-CPB and $\mathrm{CPB}+$ Levo groups, it fell in $\mathrm{CPB}$ lambs $(P<0.05)$. Blood glucose was unchanged in both $\mathrm{CPB}$ and $\mathrm{CPB}+$ Levo groups. By contrast, lactate remained elevated postoperatively in $\mathrm{CPB}$ lambs $(P<0.05)$ but was initially elevated $(P<0.001)$ and then returned to near-baseline values in $\mathrm{CPB}+\mathrm{Levo}$ lambs.

Compared with baseline, heart rate was unchanged in non$\mathrm{CPB}$ and $\mathrm{CPB}$ lambs, but fell in $\mathrm{CPB}+\mathrm{Levo}$ animals by $120 \mathrm{~min}$ after cross-clamp removal (Figure 1a; $P<0.05$ ). While $P_{\mathrm{AO}}$ was higher than baseline in non-CPB lambs (Figure 1b; $P<0.005$ ), it remained unchanged at $60 \mathrm{~min}$ after cross-clamp removal in both $\mathrm{CPB}$ and $\mathrm{CPB}+$ Levo animals $(P=0.1)$, before falling over the ensuing $180 \mathrm{~min} . P_{\mathrm{PA}}$ was also slightly higher than baseline in non-CPB lambs (Figure 1c; $P<0.05$ ), but was markedly raised at $60 \mathrm{~min}$ after cross-clamp removal in CPB and $\mathrm{CPB}+$ Levo animals $(P<0.001)$, and then decreased to nearbaseline levels by $240 \mathrm{~min}$.

CO was 29\% lower than baseline between 60 and $240 \mathrm{~min}$ after cross-clamp removal in the $\mathrm{CPB}$ group (Figure 1d; $P<$ 0.05 ) but unchanged in non- $\mathrm{CPB}$ and $\mathrm{CPB}+$ Levo lambs. However, $Q_{C A}$ fell by $25 \%$ in non-CPB (Figure 1e; $P=0.001$ ) and by $25 \%$ in $\mathrm{CPB}$ lambs $(P<0.01)$ in the same interval but preserved in the $\mathrm{CPB}+\mathrm{Levo}$ group. Consequently, whereas

Table 1. Hemodynamic and blood gas data at baseline for the three study groups

\begin{tabular}{lccc}
\hline Variable & Non-CPB controls & CPB only & CPB+Levo \\
\hline Weight $(\mathrm{kg})$ & $11.5 \pm 0.48$ & $11.6 \pm 0.89$ & $10.8 \pm 0.17$ \\
$\mathrm{pH}$ & $7.35 \pm 0.01$ & $7.34 \pm 0.01$ & $7.33 \pm 0.01$ \\
$\mathrm{PaCO}_{2}(\mathrm{~mm} \mathrm{Hg})$ & $38 \pm 2.5$ & $36 \pm 2.4$ & $39 \pm 1.2$ \\
$\mathrm{PaO}_{2}(\mathrm{~mm} \mathrm{Hg})$ & $135 \pm 8.3$ & $161 \pm 27.3$ & $125 \pm 6.4$ \\
$\mathrm{SvO}_{2}(\mathrm{~mm} \mathrm{Hg})$ & $73.8 \pm 2.6$ & $75.2 \pm 2.7$ & $71.7 \pm 2.7$ \\
$\mathrm{HR}(\mathrm{beats} / \mathrm{min})$ & $196 \pm 11.0$ & $196 \pm 11.6$ & $209 \pm 8.3$ \\
$P_{\mathrm{AO}}(\mathrm{mm} \mathrm{Hg})$ & $68 \pm 3.6$ & $70 \pm 6.8$ & $61 \pm 3.0$ \\
$P_{\mathrm{PA}}(\mathrm{mm} \mathrm{Hg})$ & $18.3 \pm 1.4$ & $19.3 \pm 1.5$ & $24.5 \pm 2.3$ \\
$\mathrm{CO}(\mathrm{ml} / \mathrm{kg} / \mathrm{min})$ & $203 \pm 12.8$ & $212 \pm 17.4$ & $169 \pm 14.9$ \\
$Q_{\mathrm{CA}}(\mathrm{ml} / \mathrm{kg} / \mathrm{min})$ & $19.5 \pm 2.5$ & $16.6 \pm 2.0$ & $18.0 \pm 2.0$ \\
\hline
\end{tabular}

$\mathrm{CO}$, cardiac output; $\mathrm{CPB}$, cardiopulmonary bypass; $\mathrm{HR}$, heart rate; Levo, levosimendan; $\mathrm{PaCO}_{2^{\prime}}$, arterial partial pressure of carbon dioxide; $\mathrm{PaO}_{2^{\prime}}$ arterial partial pressure of oxygen; $P_{\mathrm{AO}^{\prime}}$ mean aortic pressure; $P_{\mathrm{PA}^{\prime}}$ mean pulmonary artery pressure; $Q_{C A^{\prime}}$ flow in right carotid artery; $\mathrm{SvO}_{2}$, mixed venous (pulmonary artery) oxygen saturation. the $Q_{\mathrm{CA}}$-to-CO ratio fell during the study period in non-CPB lambs (Figure 1f; $P<0.05$ ), it was unchanged in both $\mathrm{CPB}$ and $\mathrm{CPB}+$ Levo animals.

\section{Magnetic Resonance Imaging}

No abnormalities were seen on conventional T1- and T2-weighted imaging in any lamb, but a single focus of $2 \mathrm{~mm}$ white matter (WM) injury in the left frontal region was identified on diffusion weighted imaging and apparent diffusion coefficient mapping in a non-CPB lamb, and a small choroid plexus hemorrhage was seen on susceptibility weighted imaging in one CPB lamb. These alterations did not specifically correlate with differences in the immunohistochemical analysis.

\section{Histology and Immunohistochemistry}

No intracerebral or intraventricular hemorrhages or major macroscopic abnormalities were identified. However, two main findings were evident on immunohistochemical analysis (Table 3 and Figure 2). Firstly, while the areal densities of anti-glial fibrillary acidic protein immunoreactive (GFAP-IR) astrocytes were similar in all groups in the gray matter (GM), the density of subcortical WM GFAP-IR astrocytes was higher in $\mathrm{CPB}$ and $\mathrm{CPB}+$ Levo lambs than in non- $\mathrm{CPB}$ animals (Table 3; $P<0.05$; Figure 2a-c). Secondly, whereas the areal density of anti-ionized calcium-binding adapter molecule 1 immunoreactive (Iba1-IR) microglia/macrophages did not differ between groups in the cortex, the overall density of Iba1-IR cells in WM was increased in $\mathrm{CPB}+$ Levo lambs compared with the non-CPB and $\mathrm{CPB}$ groups (Table 3; $P<0.05$; Figure 2d-f).

There was no difference $(P>0.05)$ between groups in the proportion of vascularity of the brain parenchyma. Extravasation of sheep serum from blood vessels into the surrounding parenchyma was evident in all groups (Figure $2 \mathrm{~g}-\mathrm{i}$ ), with the majority of serum-IR staining being in the WM. While extravasation was extensive in two of the six CPB+Levo lambs, semiquantitative scoring revealed no overall differences between groups. Rabbit anti-malondialdehyde immunoreactive (MDA-IR)

Table 2. Arterial lactate, glucose, and mixed venous oxygen saturation over time

\begin{tabular}{|c|c|c|c|c|c|}
\hline & Baseline & $60 \mathrm{~min}$ & $120 \min$ & $180 \mathrm{~min}$ & $240 \mathrm{~min}$ \\
\hline \multicolumn{6}{|l|}{$\mathrm{SvO}_{2}(\%)$} \\
\hline $\begin{array}{l}\text { Non-CPB } \\
\text { controls }\end{array}$ & $73.8 \pm 2.6$ & $74.5 \pm 1.0)$ & $74.0 \pm 2.1$ & $72.4 \pm 1.6$ & $70.4 \pm 2.4$ \\
\hline CPB & $75.2 \pm 2.7$ & $69.4 \pm 3.7^{*}$ & $65.0 \pm 4.0^{*}$ & $65.3 \pm 3.5^{*}$ & $62.1 \pm 6.5^{*}$ \\
\hline CPB+Levo & $71.7 \pm 2.7$ & $69.9 \pm 3.3$ & $65.4 \pm 4.9$ & $70.7 \pm 3.6$ & $69.5 \pm 3.5$ \\
\hline \multicolumn{6}{|c|}{ Lactate (mmol/l) } \\
\hline $\mathrm{CPB}$ & $1.8 \pm 0.3$ & $3.7 \pm 0.3^{*}$ & $3.3 \pm 0.5^{*}$ & $3.1 \pm 0.9^{*}$ & $3.8 \pm 1.5^{*}$ \\
\hline CPB+Levo & $1.8 \pm 0.1$ & $4.2 \pm 0.6^{*}$ & $3.6 \pm 0.5^{*}$ & $3.1 \pm 0.5^{*}$ & $2.4 \pm 0.3$ \\
\hline \multicolumn{6}{|c|}{ Glucose $(\mathrm{mmol} / \mathrm{l})$} \\
\hline CPB & $6.5 \pm 0.8$ & $7.6 \pm 1.7$ & $7.7 \pm 1.4$ & $7.2 \pm 1.2$ & $6.2 \pm 1.0$ \\
\hline CPB+Levo & $5.7 \pm 0.9$ & $5.3 \pm 1.3$ & $4.9 \pm 0.7$ & $4.0 \pm 0.6$ & $4.6 \pm 0.2$ \\
\hline
\end{tabular}




\section{Articles $\mid$ Namachivayam et al.}
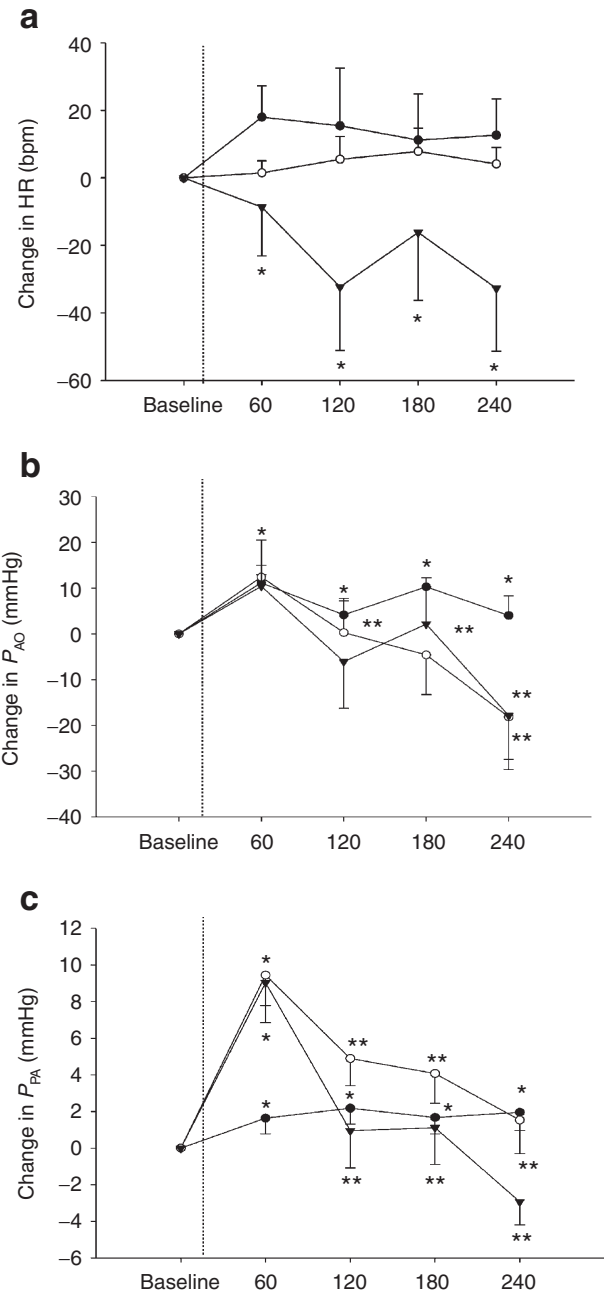

d
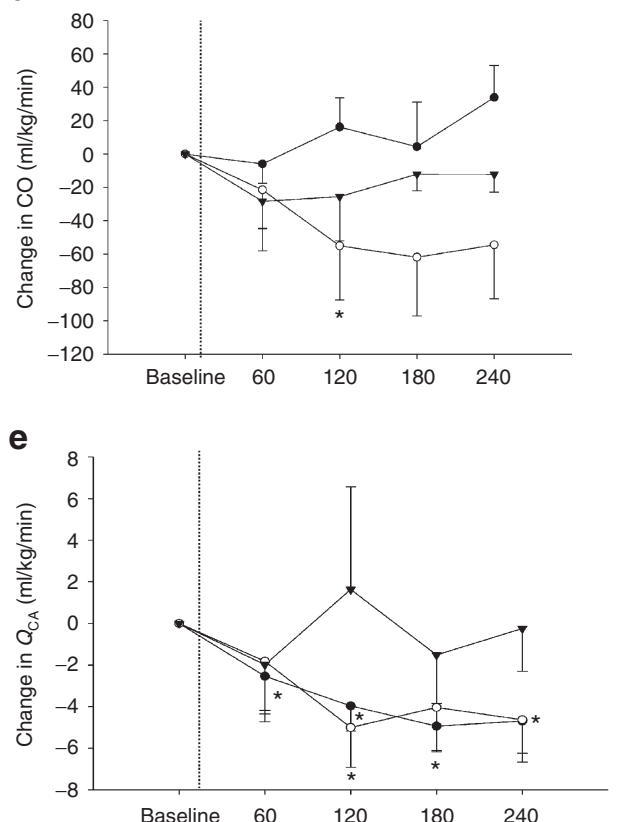

f

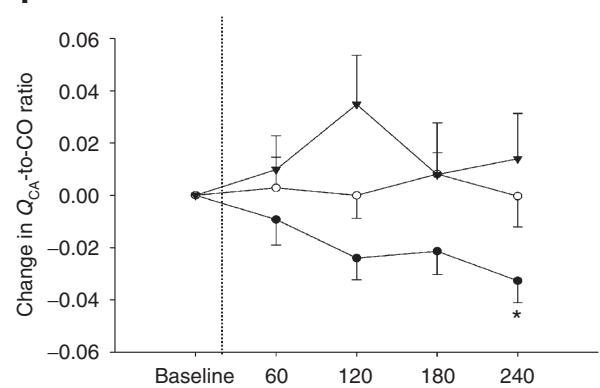

Figure 1. Hemodynamic changes relative to baseline over time. (a) Heart rate, (b) aortic pressure $\left(P_{\mathrm{AD}}\right),(\mathbf{c})$ mean pulmonary artery pressure $\left(P_{\mathrm{PA}}\right)$, (d) cardiac output (CO), (e) right carotid artery blood flow $\left(Q_{C A}\right)$, and (f) $Q_{C A}$-to-CO ratio in non-CPB (closed circle), CPB (open circle), and CPB Levo (inverted triangle) groups. Dopamine in CPB group and Levo in CPB+Levo group were started immediately after XC removal (dotted line). Baseline measurement taken before commencement of CPB. $x$-Axis represents change in minutes from XC removal and $y$-axis represents hemodynamic change relative to baseline. Mean values are shown, and error bars indicate SEM. ${ }^{*} P<0.05$ compared with baseline and ${ }^{* *} P<0.05$ compared with 60 min after $X C$ removal. $\mathrm{CPB}$, cardiopulmonary bypass; $\mathrm{HR}$, heart rate; Levo, levosimendan; $\mathrm{XC}$, cross-clamp.

Table 3. Details of brain histopathology

\begin{tabular}{|c|c|c|c|}
\hline Parameter & Non-CPB & $\mathrm{CPB}$ & CPB+Levo \\
\hline Astrocyte density cortex (cells/mm²) & $212.6 \pm 6.2$ & $196.4 \pm 12.0$ & $205.2 \pm 8.3$ \\
\hline Astrocyte density WM (cells/mm²) & $231.3 \pm 7.1$ & $286.3 \pm 18.1^{*}$ & $280.9 \pm 5.8^{*}$ \\
\hline Microglial density WM (cells/mm²) & $159.5 \pm 5.2$ & $161.8 \pm 11.4$ & $188.9 \pm 7.1^{* *}$ \\
\hline Oligodendrocyte density subcortical WM (cells/mm²) & $1,063.2 \pm 53.7$ & $1,013.3 \pm 24.0$ & $1,066.0 \pm 34.8$ \\
\hline TUNEL-positive cells in gray matter (cells/mm²) & $16.8 \pm 2.4$ & $20.5 \pm 4.8$ & $16.2 \pm 2.9$ \\
\hline TUNEL-positive cells in periventricular WM (cells $/ \mathrm{mm}^{2}$ ) & $8.7 \pm 1.5$ & $8.63 \pm 1.1$ & $7.4 \pm 1.2$ \\
\hline$\%$ of cortical parenchyma occupied by blood vessels & $2.0 \pm 0.5$ & $2.9 \pm 0.6$ & $2.5 \pm 0.3$ \\
\hline$\%$ of WM occupied by blood vessels & $3.5 \pm 0.3$ & $4.2 \pm 0.5$ & $4.2 \pm 0.6$ \\
\hline Serum-IR staining & $1.0 \pm 0.2$ & $1.5 \pm 0.2$ & $2.2 \pm 0.13$ \\
\hline
\end{tabular}

CPB, cardiopulmonary bypass; IR, immunoreactive; Levo, levosimendan; TUNEL, terminal deoxynucleotidyl transferase nick-end labeling; WM, white matter.

${ }^{*} P<0.05$ compared with non-CPB controls. ${ }^{* *} P<0.05$ compared with controls and CPB. 


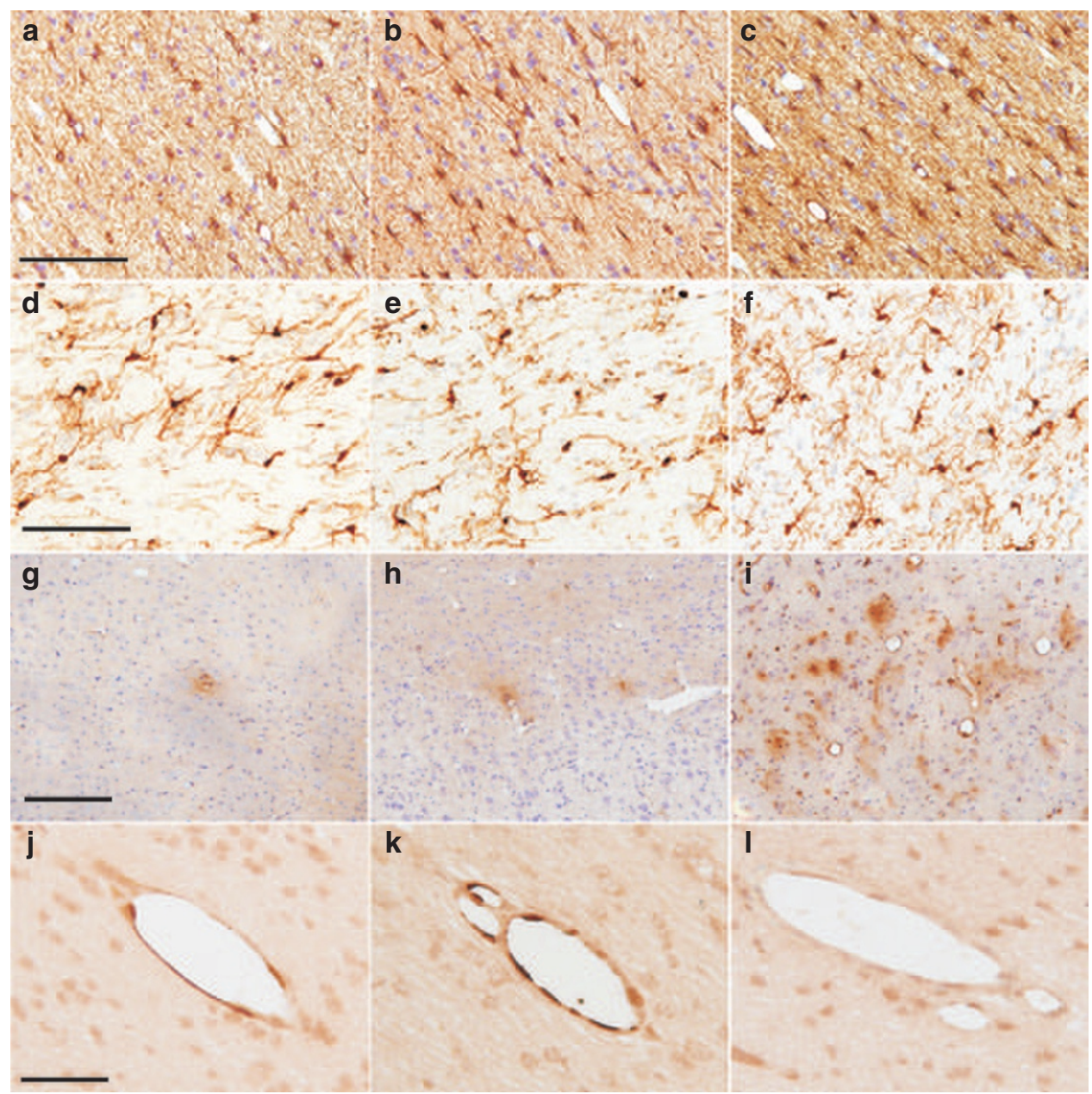

Figure 2. Brain histopathology. Left-hand column: non-CPB group. Middle column: CPB group. Right-hand column: CPB+Levo group. (a-c) GFAP-IR staining in the subcortical white matter (WM), showing increased areal density of astrocytes in the CPB (b) and CPB+Levo (c) lambs compared with non-CPB (a) lambs. (d-f) Iba-IR staining in the deep WM illustrating the extensive network of microglia present in lambs, with areal density of microglia in the CPB+Levo (f) group increased compared with non-CPB (d) and CPB lambs (e). (g-i) Sheep serum-IR showing extravasation of serum proteins in the parenchyma, with mostly small patches of extravasation in non-CPB (g) and CPB (h) groups; extensive extravasation was seen in two of the CPB+Levo animals (i). (j-I) MDA-IR staining in the endothelial cells of non-CPB (j) and CPB (k) animals. Endothelial staining was present in all CPB animals, but in only half of the animals in the non-CPB and CPB+Levo groups (I). Scale bars: $\mathbf{a}-\mathbf{i}=100 \mu \mathrm{m} ; \mathbf{j}-\mathbf{I}=50 \mu \mathrm{m}$. CPB, cardiopulmonary bypass; GFAP-IR, glial fibrillary acidic protein immunoreactive; Levo, levosimendan; MDA-IR, malondialdehyde immunoreactive.

staining (which is indicative of oxidative stress) was seen in the endothelial cells of all CPB lambs, compared with only three of six non-CPB animals and three of six $\mathrm{CPB}+$ Levo lambs (Figure 2j-1). MDA-IR was also observed in layers 5 and 6 of the cortex in all CPB lambs (Figure 3b), compared with only two of six non-CPB animals and none of the $\mathrm{CPB}+$ Levo group (Figure 3a). There was no difference $(P>0.05)$ between groups in the areal density of Olig2-IR oligodendrocytes in the subcortical or periventricular WM (Figure 3c, periventricular WM non-CBP animal). Qualitative assessment suggested that caspase3-IR apoptotic cells were present in the periventricular WM and corpus callosum, and also (though less densely) in the cortex and deep GM, predominantly the parietal and temporal lobes. Apoptosis was confirmed with terminal deoxynucleotidyl transferase nick-end labeling (TUNEL) staining (Figure 3d, WM CBP animal) and counts performed in these sections (where staining was more distinct than with
caspase3-IR) revealed no difference $(P>0.05)$ in the number of TUNEL-positive cells.

\section{DISCUSSION}

To our knowledge, this is the first study to have investigated the effects of Levo on carotid artery blood flow, brain MRI, and immunohistochemical markers of brain injury in a lamb model of infant CPB. Our results indicated that commencement of Levo infusion during weaning from CPB preserved both total $\mathrm{CO}$ and carotid artery blood flow in the early postoperative period. However, while Levo reduced a tissue marker of brain oxidative stress accompanying $\mathrm{CPB}$, it increased the density of microglia in the deep WM, which may be suggestive of mild brain injury.

The emergence of a sustained reduction in $\mathrm{CO}$ at $2 \mathrm{~h}$ after cross-clamp removal in CPB lambs (Figure 1) was consistent with development of the typical decline in CO early after $\mathrm{CPB}$. 


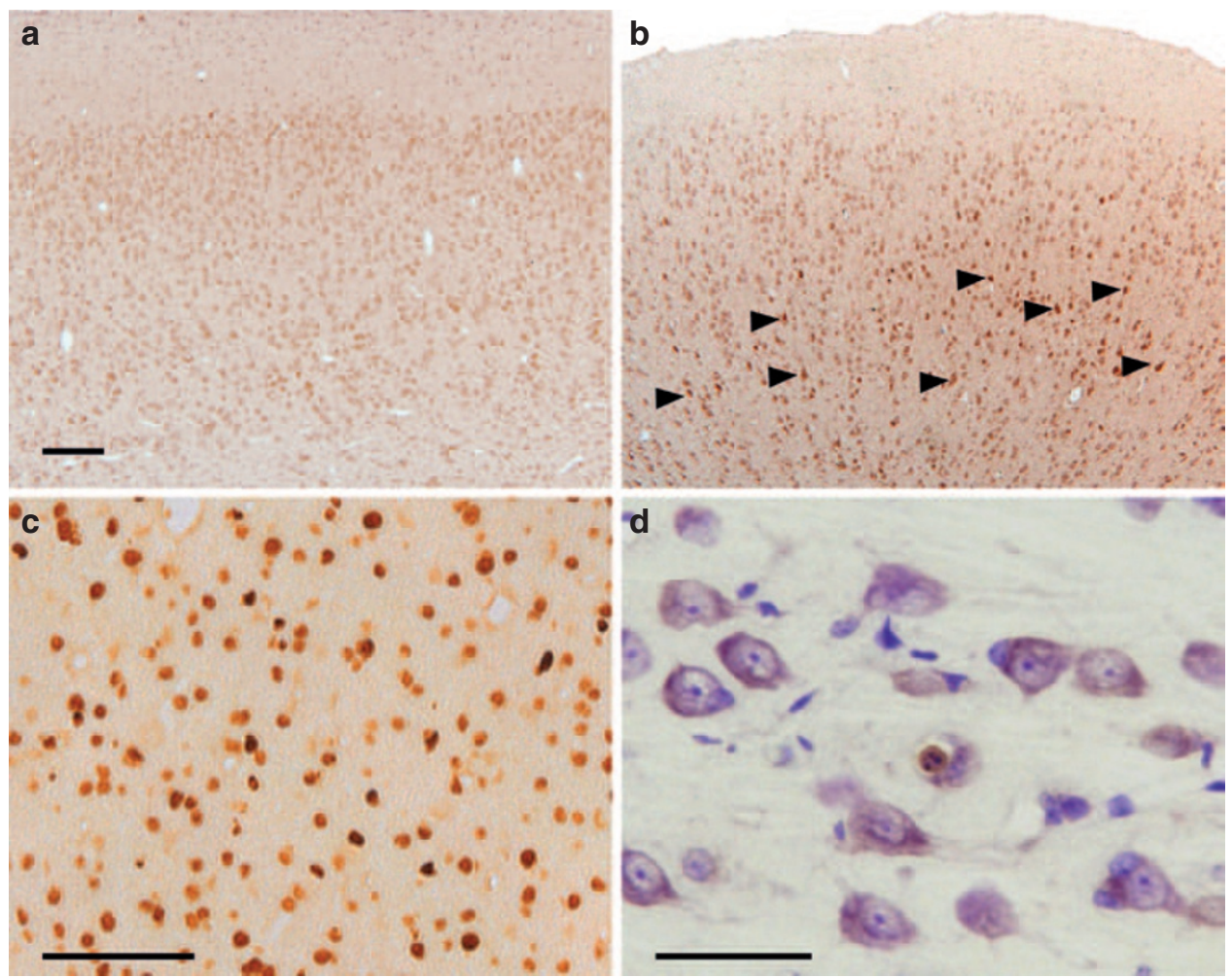

Figure 3. MDA-IR layers 5 and 6 of the cerebral cortex. (a) No staining in a CBP+Levo lamb and (b) positive staining (arrows), CPB lamb. (c) Olig-2-IR in the periventricular white matter, non-CBP lamb. (d) TUNEL staining in the periventricular white matter, CBP lamb. Scale bars: $\mathbf{a}, \mathbf{b}=200 \mu \mathrm{m} ; \mathbf{c}=100 \mu \mathrm{m}$; d $=50 \mu \mathrm{m}$. CPB, cardiopulmonary bypass; Levo, levosimendan; MDA-IR, malondialdehyde immunoreactive; TUNEL, terminal deoxynucleotidyl transferase nick-end labeling.

In this study, the CO fell by around $60 \mathrm{ml} / \mathrm{kg} / \mathrm{min}$, which is threefold that observed in our previous study in piglets (10), implying that detrimental circulatory effects of $\mathrm{CPB}$ may be more pronounced in lambs. The postoperative decrease in $\mathrm{CO}$ in CPB lambs was accompanied by a parallel fall in carotid artery blood flow (Figure 1), implying that cerebral perfusion was also reduced.

The institution of a Levo infusion at the timing of weaning from $\mathrm{CPB}$ prevented a postoperative fall in $\mathrm{CO}$, which is similar to our previous observations in piglets after $\mathrm{CPB}(10)$. In association with an unchanged $\mathrm{CO}$, postoperative carotid artery blood flow was maintained in $\mathrm{CPB}+$ Levo lambs (Figure 1), suggesting that cerebral perfusion was also preserved. While previous studies have demonstrated that Levo improves blood flow responses in the coronary $(14,15)$, renal, liver, and splanchnic circulations (16), our study is the first to investigate its effects on the brain.

$\mathrm{CPB}$ resulted in increased brain oxidative stress, which is supported by the presence of cortical and endothelial MDA-IR staining, in all CPB lambs. Infusion of Levo was associated with reduced endothelial MDA-IR staining, and the complete absence of cortical staining, suggesting a potential protective effect on oxidative injury. As well as its circulatory effects, $\mathrm{CPB}$ elicits a systemic inflammatory response that is known to adversely affect brain cellular components and processes (1719). Microglia and astrocytes are abundant in brain tissue and have a primary role in the response to inflammatory insults and in neuroprotection $(20,21)$. Both microglia and astrocytes respond to cerebral insults by acutely increasing in density, by releasing proinflammatory cytokines and free radicals that reflect and contribute to degeneration, and with the release of antioxidants and other factors that may confer neuroprotection (21). Our observation of increased astrocyte density in the WM after $\mathrm{CPB}$, which was not present in non-CPB lambs, reflects the impact of an inflammatory insult of $\mathrm{CPB}$ on the immature brain. Surprisingly, however, given that stimulation of mitochondrial ATP-sensitive $\mathrm{K}$ channels in the brain is considered to confer neuroprotection $(12,22), \mathrm{CPB}+$ Levo lambs exhibited a raised microglial density within WM that was not seen with $\mathrm{CPB}$ alone. We have previously shown a strong correlation between the intensity of microgliosis and the extent of brain injury in an ovine model of endotoxin exposure (23) suggesting a substantial role for microglial activation in the manifestation of and/or response to injury or other adverse events in this species. As the brain response was only monitored in the immediate term in this study, we cannot conclude unequivocally that injury would have developed. Phenotyping of the microglia would help to ascertain the true role of microglial changes in the CBP-Levo response. Furthermore, we note that microglial activation is not invariably associated with brain injury as demonstrated in rodent models of hypoxic ischemia $(24,25)$.

Taken together, these immunohistochemical findings suggest that the effect of Levo on brain injury patterns is not 
only heterogeneous, but maybe more complex than presently believed, which is in keeping with the recent finding that Levo did not provide neuroprotection in an animal model of global transient ischemia and hypoxia (26).

Neuronal apoptosis has been reported after CPB $(27,28)$, and Levo has been shown in animal models to have antiapoptotic effects in the myocardium and brain, through its actions on mitochondrial ATP-dependent potassium channels $(13,29,30)$. However, while apopotosis, as indicated by positive TUNEL staining, was present in all study groups, no significant difference was evident in its prevalence between groups. A potential explanation for this finding relates to the growing evidence that inhalational anesthetic agents such as isoflurane may induce neuronal apoptosis (31-34). In terms of apoptotic effects, the relatively prolonged exposure of all study groups to isoflurane in our protocol may therefore have confounded any more subtle manifestations of CPB or Levo.

Several methodological issues warrant comment. First, an important limitation of our study was the relatively short duration of postoperative monitoring $(4 \mathrm{~h})$, which undoubtedly impacted on the likelihood of seeing more established brain injury on MRI brain and ex vivo, even though gliosis and increased cytokine production (tumor necrosis factor- $\alpha$, interleukin 1, interleukin 6) are demonstrable as early as $1 \mathrm{~h}$ after an acute brain insult (35). Moreover, the typical nadir of the low output state occurs between 6 and $12 \mathrm{~h}$ after separation from $\mathrm{CPB}$. Longer term studies are therefore indicated to follow up the findings of this initial study. Second, our lamb model of CPB offered distinct advantages for the study of structural brain injury, as the immature brain of this species demonstrates similar vulnerability to early injury as humans (36) while, as in humans, lamb brains are gyrencephalic (37). Finally, our study measured carotid artery blood flow rather than cerebral blood flow directly. However, even though approximately $50 \%$ of carotid artery blood flow in the lamb is distributed to extracerebral structures, a close relationship exists between changes in carotid artery blood flow and brain blood flow measured with radioactive microspheres (38).

\section{Conclusion}

Levo prevented early postoperative falls in $\mathrm{CO}$ and carotid artery blood flow in a lamb model of infant CPB. This was in association with heterogeneous neuroglial activation and manifestation of markers of oxidative stress. The clinical significance of these findings and their underlying mechanisms warrant further investigation in a longer term model.

\section{METHODS}

\section{Surgical Preparation (All Groups)}

Eighteen Border-Leicester cross-lambs aged 3-4 wk were assigned to one of three protocols: (i) non-CPB controls $(n=6)$, (ii) CPB only ( $n$ $=6$ ), and (iii) CPB+Levo $(n=6)$. Anesthesia was induced with $4 \%$ isoflurane delivered by mask, and after tracheal intubation, maintained with inhaled isoflurane (1-3\%) and an intravenous fentanyl infusion $(20-30 \mu \mathrm{g} / \mathrm{kg} / \mathrm{h})$. Animals were ventilated using pressure-controlled ventilation (Servo Ventilator 900C, Siemens Medical Systems, Solna, Sweden), with ventilation adjusted to maintain arterial $\mathrm{Po}_{2}$ and $\mathrm{PcO}_{2}$ within physiological limits. Central temperature was monitored with a nasopharyngeal temperature probe and maintained at $37-38{ }^{\circ} \mathrm{C}$ using a heating pad and towel covering. A single dose of intravenous buprenorphine $(0.3 \mathrm{mg})$ was given for analgesia prior to sternotomy and neuromuscular blockade achieved throughout the study period with pancuronium $(0.2 \mathrm{mg} / \mathrm{kg}$ boluses $)$. Intravenous maintenance fluids $(0.45 \%$ saline with $5 \%$ dextrose, $25 \mathrm{ml} / \mathrm{h}$ ) were administered throughout the study.

Through a cut-down, a triple lumen catheter was inserted into the right femoral vein for fluid and drug administration, while a fluidfilled catheter was advanced via the right femoral artery into the descending thoracic aorta for monitoring of $P_{\mathrm{AO}}$ and blood sampling for gas analysis, and in $\mathrm{CPB}$ and $\mathrm{CPB}+\mathrm{Levo}$ groups, measurement of lactate and glucose concentrations. Through a midline neck incision, $Q_{\mathrm{CA}}$ was measured with a $4 \mathrm{~mm}$ perivascular transit-time flow probe (S-series, Transonic Systems, Ithaca, NY). The heart was exposed via a median sternotomy and suspended in a pericardial cradle. A fluidfilled catheter was placed in the main pulmonary artery for monitoring $P_{\mathrm{PA}}$ and measurement of $\mathrm{SvO}_{2}$. A 10 or $12 \mathrm{~mm}$ nonrestrictive flow probe (A-series, Transonic Systems) was placed around the ascending aorta to measure CO. In all lambs, baseline blood gas and physiologic data were collected after completion of instrumentation.

\section{Additional Procedures in CPB Groups}

An infant CPB circuit with a roller pump (Stockert CAPS, Munich, Germany) and a membrane oxygenator (Terumo SX10, Terumo Corporation, Tokyo, Japan) was primed with $500 \mathrm{ml}$ of heparinized ewe's blood, obtained just before the lamb surgery and concentrated to a hemoglobin level of $85-90 \mathrm{~g} / \mathrm{l}$. Lambs were given intravenous heparin $(300 \mathrm{IU} / \mathrm{kg})$ and placed on $\mathrm{CPB}$ at $32{ }^{\circ} \mathrm{C}$ following right atrial and aortic cannulation, with a flow rate of $150 \mathrm{ml} / \mathrm{kg} / \mathrm{min}$. After crossclamping of the aorta, crystalloid cardioplegia solution was infused into the aortic root, the heart topically cooled with ice, and cardioplegic arrest maintained by infusion of additional cardioplegia every 20 min during the cross-clamp period. The aortic cross-clamp was released after $90 \mathrm{~min}$ and lambs warmed to the baseline temperature. At the end of $\mathrm{CPB}$, modified ultrafiltration was performed (removing $50 \mathrm{ml} / \mathrm{kg}$ ultrafiltrate) and full mechanical ventilation recommenced. The total duration of $\mathrm{CPB}$, including weaning after cross-clamp removal, was approximately $2 \mathrm{~h}$. An alpha-stat blood gas strategy was used to manage blood $\mathrm{pH}$ throughout the study.

\section{Experimental Protocol and Measurements}

Heart rate, $P_{\mathrm{AO}}, P_{\mathrm{PA}}$, and $Q_{\mathrm{CA}}$ were monitored continuously throughout the study period. In $\mathrm{CPB}$ and $\mathrm{CPB}+$ Levo lambs, the aortic flow probe was removed during the period of cross-clamping, but reapplied after cessation of CPB. In CPB and CPB+Levo lambs, hemodynamics and blood gases were measured at baseline (i.e., pre-CPB) and at $60,120,180$, and $240 \mathrm{~min}$ after cross-clamp removal, with measurement of these variables at corresponding times in non-CPB lambs. During weaning from $\mathrm{CPB}$, a continuous infusion of dopamine was commenced at a rate of $5 \mu \mathrm{g} / \mathrm{kg} / \mathrm{min}$ in CPB lambs, with addition of intravenous Levo (loading dose $12.5 \mu \mathrm{g} / \mathrm{kg}$ over $10 \mathrm{~min}$, followed by a continuous infusion of $0.2 \mu \mathrm{g} / \mathrm{kg} / \mathrm{min}$ (39) for the remainder of the study period) in $\mathrm{CPB}+$ Levo lambs.

\section{Magnetic Resonance Imaging}

Following completion of the laboratory protocol, lambs underwent an MRI brain scan under anesthesia (3 Tesla Magnetom magnet, Siemens, Erlangen, Germany, Software Versions 13B and 15B). MRI sequences included axial T1, multiplanar T2, and susceptibility weighted imaging and diffusion weighted imaging with apparent diffusion coefficient mapping. All MRI scans were reviewed for the presence of hemorrhage (grade, site, and type), WM injury, deep nuclear GM injury, myelination, volume loss, and edema by a neuroradiologist (L.C.), who was blinded to any experimental details.

\section{Histology and Immunohistochemistry}

After completion of MRI scans, anesthetized lambs were returned to the laboratory where, after cannulation of both carotid arteries and euthanasia with intravenous sodium pentobarbitone $(100 \mathrm{mg} / \mathrm{kg})$, the brain was cleared of blood using heparinized Hartmann's solution, fixed by perfusion with $4 \%$ paraformaldehyde in $0.1 \mathrm{~mol} / \mathrm{l}$ phosphate buffer, and then immersed in the same fixative for a further $48 \mathrm{~h}$. 
The right cerebral hemisphere was subsequently dissected into 5 - $\mathrm{mm}$ blocks and processed to paraffin. Serial 8 - $\mu \mathrm{m}$ sections were cut from each block, with one section per block stained with hematoxylin and eosin. For each antibody, immunohistochemistry was performed on four sections taken from similar levels of the rostrocaudal extent of the forebrain (midfrontal, anterior and posterior parietal/temporal, midoccipital) with use of (i) rabbit anti-GFAP (1:500; DAKO, Glostrup, Denmark) to identify reactive astrocytes, (ii) rabbit anti-ionized calcium-binding adapter molecule 1 (Iba1; 1:500; Wako, Richmond, VA) to identify microglia and macrophages, (iii) rabbit anti-sheep whole serum (1:1,000; Sigma, St. Louis, MO) to asses blood-brain barrier integrity, and (iv) rabbit anti-MDA (1:400; Abcam, Cambridge, UK) to identify oxidative stress. Sections were also stained with Colorimetric TUNEL System (Promega, Madison, WI) to identify cell death (apoptosis and necrosis). Freefloating immunohistochemistry for caspase3 (1:2,000; Cell Signalling Technology, Danvers, MA) was performed on $40-\mu \mathrm{m}$ frozen sections cut from five blocks sampled from the rostrocaudal extent of the left cerebral hemisphere.

All histological and histochemical analyses were performed on coded slides with the observer blinded to study groups. Qualitative analysis was performed on hematoxylin and eosin sections for the presence of hemorrhages, infarcts, cystic WM lesions, and neuronal cell death, while MDA-IR in cortical layers and endothelium was also assessed. Semiquantitative analysis was performed on sections stained for whole serum-IR to assess the presence of reactivity around cerebral blood vessels, indicative of blood-brain barrier breakdown or weakening. The total number of capillaries affected across the GM and WM assessed was scored as $0=$ none, $1=1-2,2=3-10$, and $3=>10$ capillaries. For quantitative analysis, randomly selected fields of view were measured in four stained sections (for each antibody and for each brain) using an image analysis system (Image Pro Plus v4.1, Media Cybernetics, Rockville, MD). GFAP- and Iba1-positive cells were counted in three fields in the cortex (layers 2-4) and two fields in each of the deep and subcortical WM and expressed as cells $/ \mathrm{mm}^{2}$. TUNEL-positive cells were counted in the periventricular WM (in the same regions as for the immunohistochemical staining) and in the deep GM. Vascularization was assessed by point counting to determine the percentage of cortex and WM occupied by blood vessels in GFAP-IR sections.

\section{Statistical Analysis}

Data were analyzed using SigmaPlot version 11 for windows (SyStat Software, San Jose, CA), preceded by logarithmic transformation where data had a nonnormal distribution. Baseline hemodynamic and blood gas variables, as well as histological and immunohistochemical data, were analyzed using one-way ANOVA, with post hoc analysis performed using the Bonferroni correction or Tukey's test. Changes in hemodynamic and blood gas data were analyzed using repeated measures one-way ANOVA, with specific within-group comparisons between time points evaluated by partitioning the sums of squares into individual degrees of freedom (40). Results are expressed as mean \pm SEM, and $P<0.05$ was considered significant.

Experiments were approved by the Murdoch Childrens Research Institute Animal Ethics Committee and conformed with guidelines of the National Health and Medical Research Council of Australia.

\section{ACKNOWLEDGMENTS}

We thank Magdy Sourial and Shane Osterfield of The Royal Children's Hospital Large Animal Facility for technical assistance with experimental studies, and Michael Kean of the Royal Children's Hospital Department of Medical Imaging for performing and coordinating MRI scans.

\section{STATEMENT OF FINANCIAL SUPPORT}

This work was supported by a Grant-in-Aid from the National Heart Foundation of Australia and the Victorian Government's Operational Infrastructure Support Program.

Disclosure: The authors have no other financial ties or conflicts of interest to declare.

\section{REFERENCES}

1. Miatton M, De Wolf D, François K, Thiery E, Vingerhoets G. Neuropsychological performance in school-aged children with surgically corrected congenital heart disease. J Pediatr 2007;151:73-8, 78.e1.

2. Bellinger DC, Wypij D, duPlessis AJ, et al. Neurodevelopmental status at eight years in children with dextro-transposition of the great arteries: the Boston Circulatory Arrest Trial. J Thorac Cardiovasc Surg 2003;126:138596.

3. Snookes SH, Gunn JK, Eldridge BJ, et al. A systematic review of motor and cognitive outcomes after early surgery for congenital heart disease. Pediatrics 2010;125:e818-27.

4. Shillingford AJ, Glanzman MM, Ittenbach RF, Clancy RR, Gaynor JW, Wernovsky G. Inattention, hyperactivity, and school performance in a population of school-age children with complex congenital heart disease. Pediatrics 2008;121:e759-67.

5. Majnemer A, Limperopoulos C, Shevell M, Rosenblatt B, Rohlicek C, Tchervenkov C. Long-term neuromotor outcome at school entry of infants with congenital heart defects requiring open-heart surgery. J Pediatr 2006;148:72-7.

6. Kaltman JR, Andropoulos DB, Checchia PA, et al. Report of the pediatric heart network and national heart, lung, and blood institute working group on the perioperative management of congenital heart disease. Circulation 2010;121:2766-72.

7. Miller SP, McQuillen PS, Hamrick S, et al. Abnormal brain development in newborns with congenital heart disease. N Engl J Med 2007;357:1928-38.

8. Andropoulos DB, Hunter JV, Nelson DP, et al. Brain immaturity is associated with brain injury before and after neonatal cardiac surgery with highflow bypass and cerebral oxygenation monitoring. J Thorac Cardiovasc Surg 2010;139:543-56.

9. Hoffman TM, Wernovsky G, Atz AM, et al. Efficacy and safety of milrinone in preventing low cardiac output syndrome in infants and children after corrective surgery for congenital heart disease. Circulation 2003;107:9961002.

10. Stocker CF, Shekerdemian LS, Nørgaard MA, et al. Mechanisms of a reduced cardiac output and the effects of milrinone and levosimendan in a model of infant cardiopulmonary bypass. Crit Care Med 2007;35:252-9.

11. Shake JG, Peck EA, Marban E, et al. Pharmacologically induced preconditioning with diazoxide: a novel approach to brain protection. Ann Thorac Surg 2001;72:1849-54.

12. Shimizu K, Lacza Z, Rajapakse N, Horiguchi T, Snipes J, Busija DW. MitoK(ATP) opener, diazoxide, reduces neuronal damage after middle cerebral artery occlusion in the rat. Am J Physiol Heart Circ Physiol 2002;283:H1005-11.

13. Pollesello P, Papp Z. The cardioprotective effects of levosimendan: preclinical and clinical evidence. J Cardiovasc Pharmacol 2007;50:257-63.

14. Michaels AD, McKeown B, Kostal M, et al. Effects of intravenous levosimendan on human coronary vasomotor regulation, left ventricular wall stress, and myocardial oxygen uptake. Circulation 2005;111:1504-9.

15. Bowman P, Haikala H, Paul RJ. Levosimendan, a calcium sensitizer in cardiac muscle, induces relaxation in coronary smooth muscle through calcium desensitization. J Pharmacol Exp Ther 1999;288:316-25.

16. Pagel PS, Hettrick DA, Warltier DC. Influence of levosimendan, pimobendan, and milrinone on the regional distribution of cardiac output in anaesthetized dogs. Br J Pharmacol 1996;119:609-15.

17. Abbott NJ. Inflammatory mediators and modulation of blood-brain barrier permeability. Cell Mol Neurobiol 2000;20:131-47.

18. Cavaglia M, Seshadri SG, Marchand JE, Ochocki CL, Mee RB, Bokesch PM. Increased transcription factor expression and permeability of the blood brain barrier associated with cardiopulmonary bypass in lambs. Ann Thorac Surg 2004;78:1418-25.

19. Joshi B, Brady K, Lee J, et al. Impaired autoregulation of cerebral blood flow during rewarming from hypothermic cardiopulmonary bypass and its potential association with stroke. Anesth Analg 2010;110:321-8.

20. Ransohoff RM, Brown MA. Innate immunity in the central nervous system. J Clin Invest 2012;122:1164-71. 
21. Singh S, Swarnkar S, Goswami P, Nath C. Astrocytes and microglia: responses to neuropathological conditions. Int J Neurosci 2011;121:589-97.

22. Yamada K, Ji JJ, Yuan H, et al. Protective role of ATP-sensitive potassium channels in hypoxia-induced generalized seizure. Science 2001;292:1543-6.

23. Duncan JR, Cock ML, Suzuki K, Scheerlinck JP, Harding R, Rees SM. Chronic endotoxin exposure causes brain injury in the ovine fetus in the absence of hypoxemia. J Soc Gynecol Investig 2006;13:87-96.

24. Wang X, Stridh L, Li W, et al. Lipopolysaccharide sensitizes neonatal hypoxic-ischemic brain injury in a MyD88-dependent manner. J Immunol 2009;183:7471-7.

25. Doverhag C, Hedtjärn M, Poirier F, et al. Galectin-3 contributes to neonatal hypoxic-ischemic brain injury. Neurobiol Dis 2010;38:36-46.

26. Roehl AB, Zoremba N, Kipp M, et al. The effects of levosimendan on brain metabolism during initial recovery from global transient ischaemia/ hypoxia. BMC Neurol 2012;12:81.

27. Sato Y, Laskowitz DT, Bennett ER, Newman MF, Warner DS, Grocott HP. Differential cerebral gene expression during cardiopulmonary bypass in the rat: evidence for apoptosis? Anesth Analg 2002;94:1389-94, table of contents.

28. Ditsworth D, Priestley MA, Loepke AW, et al. Apoptotic neuronal death following deep hypothermic circulatory arrest in piglets. Anesthesiology 2003;98:1119-27.

29. Parissis JT, Andreadou I, Bistola V, Paraskevaidis I, Filippatos G, Kremastinos DT. Novel biologic mechanisms of levosimendan and its effect on the failing heart. Expert Opin Investig Drugs 2008;17:1143-50.

30. Cengiz SL, Erdi MF, Tosun M, et al. Beneficial effects of levosimendan on cerebral vasospasm induced by subarachnoid haemorrhage: an experimental study. Brain Inj 2010;24:877-85.
31. Davidson AJ. Anesthesia and neurotoxicity to the developing brain: the clinical relevance. Paediatr Anaesth 2011;21:716-21.

32. Brambrink AM, Evers AS, Avidan MS, et al. Isoflurane-induced neuroapoptosis in the neonatal rhesus macaque brain. Anesthesiology 2010;112: $834-41$.

33. Istaphanous GK, Loepke AW. General anesthetics and the developing brain. Curr Opin Anaesthesiol 2009;22:368-73.

34. Istaphanous GK, Ward CG, Nan X, et al. Characterization and quantification of isoflurane-induced developmental apoptotic cell death in mouse cerebral cortex. Anesth Analg 2013;116:845-54.

35. Feuerstein GZ, Liu T, Barone FC. Cytokines, inflammation, and brain injury: role of tumor necrosis factor-alpha. Cerebrovasc Brain Metab Rev 1994;6:341-60.

36. Duncan JR, Cock ML, Scheerlinck JP, et al. White matter injury after repeated endotoxin exposure in the preterm ovine fetus. Pediatr Res 2002;52:941-9.

37. Traystman RJ. Animal models of focal and global cerebral ischemia. ILAR J 2003;44:85-95.

38. van Bel F, Roman C, Klautz RJ, Teitel DF, Rudolph AM. Relationship between brain blood flow and carotid arterial flow in the sheep fetus. Pediatr Res 1994;35:329-33.

39. Nieminen MS, Akkila J, Hasenfuss G, et al. Hemodynamic and neurohumoral effects of continuous infusion of levosimendan in patients with congestive heart failure. J Am Coll Cardiol 2000;36:1903-12.

40. Snedecor G, Cochran W. Statistical Methods. 7th edn. Ames, IA: Iowa State University Press, 1980:350-2. 